\title{
Fatores preditivos da transfusão de concentrado de hemácias em pacientes submetidos a cirurgias eletivas do aparelho digestivo: uma análise interinstitucional
}

\author{
Predictive factors associated with packed red cell transfusions in patients submitted to elective \\ surgery of the digestive tract: an inter-institutional analysis
}

Antonio Sergio Barcala Jorge ${ }^{l}$

Marco Aurélio Severo da Silva ${ }^{2}$

Jason Costa Pereira Júnior ${ }^{2}$

Camila Xavier e Silva

Leonel Ribeiro Silva

Marcos Sílvio Gomes ${ }^{3}$

Paulo Rogério Ferreti Bonan
O objetivo desse estudo foi compreender os fatores determinantes da prática transfusional de concentrado de hemácias em cirurgias eletivas do aparelho digestivo em quatro unidades hospitalares em Montes Claros, Minas Gerais, Brasil. Foi realizado um estudo descritivo, analitico e retrospectivo através do levantamento de dados em prontuários clínicos de quatro unidades hospitalares de onde foram catalogados os dados de pacientes a partir de junho de 2007 a dezembro de 2008, referentes às cirurgias citadas. Os resultados evidenciaram 81 casos, sendo $42 \%$ do gênero feminino e 58\% do masculino. A idade média foi de 55,5 anos ( $\pm 19,11$ anos). As enfermidades de base reportadas mais comuns foram os processos neoplásicos (42\%). Desses 81 pacientes, 38 (47\%) foram hemotransfundidos no pré-operatório imediato, 28 (18\%) foram transfundidos no transcirúrgico e 37 (45,7\%) no pósoperatório imediato. Na análise univariada houve diferenças entre a prática transfusional pré-cirúrgica interinstitucional. Na fase cirúrgica houve relação entre a prática transfusional com a citação de motivos, fator RH (Rhesus), prática institucional e doença de base. Na fase pós-cirúrgica não foram demonstradas relações. Em todas as fases, não houve associação da prática transfusional com os tipos de procedimentos cirúrgicos, com o indice ASA (American Society of Anesthesiology) e valores de hemoglobina e hematócrito. Concluindo, esse estudo revelou que a prática transfusional foi heterogênea entre instituições hospitalares, careceu de valores laboratoriais especificos, relacionou-se com a patologia de base mas não com o tipo de procedimento cirúrgico e foi norteada pelo critério de escolha do profissional.

Descritores: Procedimentos cirúrgicos do sistema digestório; Transfusão de eritrócitos; Transfusão de componentes sanguíneos

Universidade Estadual de Montes Claros - Unimontes - Montes Claros (MG), Brasil.

${ }^{I}$ Departamento de Cirurgia, Universidade Estadual de Montes Claros - Unimontes - Montes Claros (MG), Brasil.

${ }^{2}$ Acadêmico, $11^{\circ}$ periodo do Curso Médico, Universidade Estadual de Montes Claros - Unimontes - Montes Claros (MG), Brasil.

${ }^{3}$ Acadêmica, $5^{\circ}$ período do Curso Médico, Universidade Estadual de Montes Claros - Unimontes - Montes Claros (MG), Brasil.

${ }^{4}$ Acadêmico, $4^{\circ}$ período do Curso Médico, Universidade Estadual de Montes Claros - Unimontes - Montes Claros (MG), Brasil.

${ }^{5}$ Programa de Pós-graduação Stricto Sensu em Ciências da Saúde, Universidade Estadual de Montes Claros - Unimontes - Montes Claros $(M G)$, Brasil.

Conflito de interesse: sem conflito de interesse

Correspondência: Paulo Rogério Ferreti Bonan

Rua Helena de Paula Fraga, 867 - Major Prates

39403-263 - Montes Claros (MG), Brasil.

Tel.: (55 38) 3213-5759

E-mail:pbonan@yahoo.com 


\section{Introdução}

A perda sanguínea significativa que ocorre durante um ato operatório tem como principal consequência o estabelecimento de um quadro de anemia aguda. Com isso, há uma inadequada liberação de oxigênio nos tecidos, logo, hipóxia tecidual. A terapêutica geralmente empregada nesses casos é a transfusão de concentrado de hemácias (TCH) a fim aumentar a capacidade de transporte de oxigênio em pacientes agudamente anêmicos. ${ }^{(1)}$ Todavia, estratégias mais restritivas têm sido empregadas no intuito de minorar as práticas transfusionais, reduzindo custo e morbimortalidade advindas de seu emprego. ${ }^{(2)}$ Existem vários estudos sobre TCH tentando estabelecer a relação de riscos versus benefícios, seu uso criterioso e racional, bem como sua real necessidade nos diversos procedimentos cirúrgicos. Os valores hematológicos que servem de "gatilho transfusional" não são reconhecidamente consensuais e as TCH são notadamente variáveis. ${ }^{(2-5)}$

Esses dados parecem corroborar a plasticidade que o organismo humano tem para compensar a perda sanguínea e suprir a dificuldade no transporte de oxigênio. Isso sugere que níveis de hemoglobina e hematócrito menores que os encontrados no ato operatório são perfeitamente aceitáveis, sem haver o emprego da TCH. ${ }^{(6,7)}$ Em adição, o uso de procedimentos alternativos à transfusão empregados no pré e transcirúrgico, como recuperação intraoperatória de células, hemodiluição com cristaloides, aumento da oferta de oxigênio administrado, utilização de eritropoetina, suplemento de ferro e vitamina $B_{12}$, tem melhorado o efeito da perda sanguínea, não resultando em discrepâncias ou alterações em relação à hipóxia, hipotensão ou arritmia. ${ }^{(6-10)}$

Observa-se também que há grande variabilidade intra e interinstitucional na prática da TCH quando da realização de procedimentos cirúrgicos semelhantes como reportados em cirurgias de bypass cardíaco, síndrome aguda coronariana e ortopédicas. ${ }^{(11-14)}$ Ao mesmo tempo, a documentação em prontuários referente à solicitação e acompanhamento dos efeitos colaterais das TCH é muitas vezes escassa. ${ }^{(4,14)}$ Em virtude da escassez de trabalhos na literatura que lidem com a variabilidade da prática da $\mathrm{TCH}$ interinstitucional em cirurgias eletivas do aparelho digestivo no Brasil e a necessidade de se compreender os fatores preditivos para a prática de $\mathrm{TCH}$ nessa área, foi objetivo desse estudo compreender os fatores determinantes da prática de $\mathrm{TCH}$ em cirurgias eletivas do aparelho digestivo em quatro unidades hospitalares em Montes Claros, Minas Gerais, Brasil.

\section{Métodos}

Foi realizado um estudo descritivo, analítico e retrospectivo através do levantamento de dados em prontuários clínicos das quatro unidades hospitalares com unidades transfusionais distintas, da cidade de Montes Claros, que possui 350.000 habitantes, situada no norte do estado de Minas Gerais. No período de junho de 2007 a dezembro de 2008 , foram catalogados dados de pacientes a partir de prontuários clínicos referentes às cirurgias eletivas do aparelho digestivo. A coleta de informações baseou-se em formulários que foram preenchidos através dos serviços de arquivamento das unidades de saúde mencionadas.

Foram incluídos nesse estudo prontuários dos pacientes submetidos a cirurgias eletivas no aparelho digestivo que receberam ou não TCH durante o período pré, trans e pós-cirúrgico. Foram excluídos os prontuários de pacientes submetidos a procedimentos cirúrgicos em multissistemas. As variáveis consideradas foram informações sobre a unidade de atendimento, status do paciente antes do procedimento cirúrgico (idade, gênero, índice ASA (American Society of Anesthesiology), histórico médico pregresso e atual, distúrbios de coagulação), dados cirúrgicos (patologia de base, tipo de intervenção, exame laboratoriais, quadro hemorrágico, quadros de instabilidade, intercorrências, no trans e pósoperatório), características da prática transfusional (realização, motivos expressos, fatores $\mathrm{ABO}$ e RH (Rhesus) e consentimento esclarecido. Os dados obtidos foram tabulados em dois bancos de dados no software SPSS 15.0 (Statistical Package for the Social Sciences, Chicago, Estados Unidos da América) sendo feitas análises estatísticas descritivas e testes qui-quadrado, Likelihood e análise univariada linear de variância.

Este estudo foi conduzido de acordo com os preceitos determinados pela resolução 196/88 do Conselho Nacional de Saúde do Ministério da Saúde. Os responsáveis legais pelas instituições de saúde tomaram conhecimento da importância do estudo assinando termo de consentimento, após informação, para autorizar o desenvolvimento da pesquisa. A presente proposição de trabalho foi submetida ao Comitê de Ética em Pesquisa da Universidade Estadual de Montes Claros e aprovada sob o $\mathrm{n}^{\circ} 1033 / 08$.

\section{Resultados}

Do período de junho de 2007 a dezembro de 2008, 81 prontuários foram selecionados para a realização desse estudo, preenchendo os critérios de inclusão. Esses 81 casos correspondiam a 34 pacientes ( $42 \%$ ) do gênero feminino e 47 (58\%) do gênero masculino. A idade média desses pacientes foi de 55,5 anos ( $\pm 19,11$ anos). As enfermidades de base reportadas foram 34 (42\%) processos neoplásicos, 18 (22,2\%) processos infecciosos e inflamatórios, $11(13,6 \%)$ estenoses, obstruções e protrusões, nove $(11,1 \%)$ processos patológicos de natureza distinta dos citados e nove $(11,1 \%)$ patologias vasculares. Os valores médios do nível mais baixo de hemoglobina apresentados pelos pacientes no período précirúrgico imediato foram de $9,77 \mathrm{~g} / \mathrm{dl}( \pm 2,07 \mathrm{~g} / \mathrm{dl})$. A mediana dos valores de hemoglobina foi de $9,7 \mathrm{~g} / \mathrm{dl}$ com o valor mínimo 
de $6,10 \mathrm{~g} / \mathrm{dl}$ e o máximo de $15 \mathrm{~g} / \mathrm{dl}$. Os valores do hematócrito médio mais baixo antes da cirurgia foram de $0,31( \pm 0,059)$. A mediana dos valores do hematócrito foi de 0,30 com o valor

Tabela 1. Relações percentuais entre a prática da TCH (Transfusão de Concentrado de Hemácias) prévia a cirurgia e as outras variáveis categóricas afins $(n=81)$

\begin{tabular}{|c|c|c|c|}
\hline \multirow{2}{*}{ Variáveis } & \multicolumn{2}{|c|}{ TCH pré-operatória } & \multirow{2}{*}{$\begin{array}{l}\text { Valor de } \\
\qquad p\end{array}$} \\
\hline & $\begin{array}{c}\operatorname{Sim} \\
\mathrm{n}(\%)\end{array}$ & $\begin{array}{l}\text { Não } \\
\text { n (\%) }\end{array}$ & \\
\hline \multicolumn{4}{|l|}{ Gênero } \\
\hline Feminino & $14(36,8)$ & $20(46,5)$ & 0,379 \\
\hline Masculino & $24(63,2)$ & $23(53,5)$ & \\
\hline \multicolumn{4}{|l|}{ Idade } \\
\hline Até 43 anos & $9(23,7)$ & $11(25,6)$ & 0,980 \\
\hline De 44 a 56 anos & $11(28,9)$ & $12(27,9)$ & \\
\hline Acima de 56 anos & $18(47,4)$ & $20(46,5)$ & \\
\hline \multicolumn{4}{|l|}{ Tipo sanguíneo } \\
\hline A & $10(26,3)$ & $20(46,5)$ & \\
\hline B & $4(10,5)$ & $3(7,0)$ & 0,306 \\
\hline $\mathrm{AB}$ & $1(2,7)$ & $1(2,3)$ & \\
\hline $\mathrm{O}$ & $23(60,5$ & $19(44,2)$ & \\
\hline \multicolumn{4}{|l|}{ Fator RH } \\
\hline Positivo & $34(89,5)$ & $38(88,4)$ & 0,875 \\
\hline Negativo & $4(10,5)$ & $5(11,6)$ & \\
\hline \multicolumn{4}{|l|}{ ASA } \\
\hline 1 e 2 & $16(42,1)$ & $24(55,8)$ & \\
\hline 3 e 4 & $8(21,1)$ & $12(27,9)$ & 0,109 \\
\hline Não Informado & $14(36,8)$ & $7(16,3)$ & \\
\hline \multicolumn{4}{|l|}{ Instituição } \\
\hline 1 & $11(28,9)$ & $2(4,7)$ & \\
\hline 2 & $14(36,8)$ & $23(53,5)$ & $0,009 *$ \\
\hline 3 & $6(15,8)$ & $4(9,3)$ & \\
\hline 4 & $7(18,5)$ & $14(32,5)$ & \\
\hline \multicolumn{4}{|l|}{ Doença de Base } \\
\hline Neoplasias & $12(31,6)$ & $22(51,2)$ & \\
\hline Processos inflamatórios/infecciosos & $9(23,7)$ & $9(20,9)$ & 0,308 \\
\hline Estenoses/obstruções/protrusões & $6(15,8)$ & $6(13,9)$ & \\
\hline Processos vasculares & $6(15,8)$ & $3(7,0)$ & \\
\hline $\begin{array}{l}\text { Processos patológicos de outra } \\
\text { natureza }\end{array}$ & $5(13,1)$ & $3(7,0)$ & \\
\hline \multicolumn{4}{|l|}{ Valores de Hemoglobina mais baixos } \\
\hline Menor ou igual a $8 \mathrm{~g} / \mathrm{dl}$ & $6(15,8)$ & $3(7,0)$ & 0,379 \\
\hline Maior que $8 \mathrm{~g} / \mathrm{dl}$ & $19(50)$ & $21(49,0)$ & \\
\hline Dado ausente & $13(34,2)$ & $19(44,0)$ & \\
\hline \multicolumn{4}{|l|}{ Valores de Hematócrito mais baixos } \\
\hline Menor ou igual a 0,3 & $17(44,8)$ & $10(23,3)$ & 0,094 \\
\hline Maior que 0,3 & $13(34,2)$ & $17(39,5)$ & \\
\hline Dado ausente & $8(21,1)$ & $16(37,2)$ & \\
\hline \multicolumn{4}{|l|}{ Distúrbio de coagulação } \\
\hline Não & $36(94,7)$ & $11(26,3)$ & 0,748 \\
\hline $\operatorname{Sim}$ & $2(5,3)$ & $27(73,7)$ & \\
\hline \multicolumn{4}{|l|}{ Citação de motivo pré-cirúrgico } \\
\hline $\operatorname{Sim}$ & $11(26,3)$ & $3(7,0)$ & $0,008^{*}$ \\
\hline Não & $27(73,7)$ & $40(93,0)$ & \\
\hline \multicolumn{4}{|l|}{ Consentimento } \\
\hline Não & $32(84,2)$ & $38(88,4)$ & 0,586 \\
\hline $\operatorname{Sim}$ & $6(15,8)$ & $5(11,6)$ & \\
\hline
\end{tabular}

* $p$ estatístico significativo $\leq 0,05$ mínimo de 0,21 e o máximo de 0,40 . Dos 81 prontuários, 50 $(61,72 \%)$ traziam os valores de hemoglobina pré-cirúrgica e $58(71,6 \%)$ os valores de hematócrito pré-cirúrgico expressos por escrito ou determinados em exames. Os valores médios para o índice ASA foram 2,3 $( \pm 0,81)$ e expressos em 60 (74\%) prontuários. Distúrbios da coagulação foram reportados em cinco casos. Dos 81 pacientes, $38(47 \%)$ receberam TCH no pré-operatório imediato. Desses, sete $(18 \%)$ foram retransfundidos no transcirúrgico e 12 (31,6\%) no pós-operatório imediato. Vinte e sete pacientes transfundidos no préoperatório não apresentaram justificativa para a transfusão no prontuário. Das justificativas para a prática da $\mathrm{TCH}$ relataram-se 12 alegações de anemia (valor médio de hemoglobina $=9,38 \mathrm{~g} / \mathrm{dl}( \pm 2,08 \mathrm{~g} / \mathrm{dl}))$, uma de choque hipovolêmico e outra de hemorragia digestiva alta. Quando da realização da análise univariada linear de variância, relacionando os valores de hemoglobina e hematócrito com a TCH pré-operatória, os $p$ estatísticos foram 0,076 e 0,164 , respectivamente. Não houve associação estatística entre a prática de TCH com valores de hemoglobina inferiores ou iguais a $8 \mathrm{~g} / \mathrm{dl} \mathrm{e}$ hematócrito inferior a 0,30 . As relações entre a prática da TCH prévia a cirurgia e as outras variáveis pré-cirúrgicas categóricas correlatas se encontram na Tabela 1. Das variáveis pré-cirúrgicas estudadas, apenas a instituição hospitalar e a citação de motivo tiveram relação com a prática de $\mathrm{TCH}$ ( $\mathrm{p}=0,009 \mathrm{e} \mathrm{p}=0,008$, respectivamente).

Os procedimentos cirúrgicos empregados foram $51(63 \%)$ ressecções e enucleações, $16(19,2 \%)$ plastias, reconstruções, derivações e desconexões, nove $(11,1 \%)$ cirurgias exploratórias e cinco $(6,2 \%)$ cauterizações e escleroses. Durante o transcirúrgico, 28 (34,6\%) pacientes receberam TCH. Foram relatadas cinco intercorrências transcirúrgicas que justificariam o procedimento transfusional, sendo três relatos de choque hipovolêmico ou quadro hemorrágico e dois de dificuldades técnicas durante o ato operatório. Não houve associação estatística entre a prática de TCH com valores de hemoglobina inferiores ou iguais a 8 $\mathrm{g} / \mathrm{dl}$ e hematócrito inferior ou igual a $0,30 \%$ no pré-cirúrgico imediato. A Tabela 2 descreve as relações entre a prática da TCH no transcirúrgico e as variáveis categóricas correlatas. Das variáveis estudadas no transcirúrgico, o fator $\mathrm{Rh}$ $(\mathrm{p}=0,037)$, a instituição $(\mathrm{p}=0,001)$, a doença de base (neoplasia) $(p=0,001)$ e a citação de motivos $(p=0,031)$ estiveram relacionadas com a prática de $\mathrm{TCH}$.

Quanto aos procedimentos de TCH pós-cirúrgicos, 37 $(45,7 \%)$ pacientes receberam o concentrado de hemácias. Os valores do nível médio mais baixo de hemoglobina no póscirúrgico foram de $9,39 \mathrm{~g} / \mathrm{dl}( \pm 2,25 \mathrm{~g} / \mathrm{dl})$ e do hematócrito, de $0,28( \pm 0,06)$. A mediana dos valores de hemoglobina foi de $9,8 \mathrm{~g} / \mathrm{dl}$ com o valor mínimo de $4,60 \mathrm{~g} / \mathrm{dl}$ e o máximo de 14,10 $\mathrm{g} / \mathrm{dl}$. A mediana dos valores do hematócrito foi de $0,28 \mathrm{com}$ o valor mínimo de 0,15 e o máximo de 0,45 . Dos 81 prontuários, $48(59,2 \%)$ traziam os valores de hemoglobina no pós-cirúrgico e 60 (74,1\%), os valores de hematócrito. Houve 
três citações de quadros hemorrágicos pós-cirúrgicos, 15 relatos de necessidade de internação pós-cirúrgica em centros de terapia intensiva e de 11 casos de óbito no póscirúrgico. Quando da realização da análise univariada linear de variância, relacionando os valores de hemoglobina e hematócrito como a prática transfusional pós-operatória, os $p$ estatísticos obtidos foram 0,801 e 0,83 , respectivamente. Não houve registros em prontuário de complicações pós-

Tabela 2. Relações entre a prática da TCH (Transfusão de Concentrado de Hemácias) transcirúrgica e as outras variáveis categóricas afins $(\mathrm{n}=81)$

\begin{tabular}{|c|c|c|c|}
\hline \multirow{2}{*}{ Variáveis } & \multicolumn{2}{|c|}{ TCH Pré-operatória } & \multirow{2}{*}{$\begin{array}{l}\text { Valor } \mathrm{d} \\
\quad p\end{array}$} \\
\hline & $\begin{array}{l}\operatorname{Sim} \\
\mathrm{n}(\%)\end{array}$ & $\begin{array}{l}\text { Não } \\
\mathrm{n}(\%)\end{array}$ & \\
\hline \multicolumn{4}{|l|}{ Gênero } \\
\hline Feminino & $11(39,3)$ & $23(43,4)$ & 721 \\
\hline Masculino & $17(60,7)$ & $30(56,6)$ & \\
\hline \multicolumn{4}{|l|}{ Idade } \\
\hline Até 43 anos & $4(14,3)$ & $16(30,2)$ & 0,142 \\
\hline De 44 a 56 anos & $7(25,0)$ & $16(30,2)$ & \\
\hline Acima de 56 anos & $17(60,7)$ & $21(39,6)$ & \\
\hline \multicolumn{4}{|l|}{ Tipo sanguíneo } \\
\hline A & $8(28,6)$ & $22(41,5)$ & \\
\hline B & $1(3,6)$ & $6(11,3)$ & 0,287 \\
\hline $\mathrm{AB}$ & $1(3,6)$ & $1(1,9)$ & \\
\hline $\mathrm{O}$ & $18(64,2)$ & $24(45,3)$ & \\
\hline \multicolumn{4}{|l|}{ Fator RH } \\
\hline Positivo & $22(78,6)$ & $50(94,3)$ & $0,037^{*}$ \\
\hline Negativo & $6(21,4)$ & $3(5,7)$ & \\
\hline \multicolumn{4}{|l|}{ Instituição } \\
\hline 1 & $1(3,6)$ & $12(22,7)$ & \\
\hline 2 & $9(32,1)$ & $28(52,8)$ & $0,001 *$ \\
\hline 3 & $4(14,3)$ & $6(11,3)$ & \\
\hline 4 & $14(50,0)$ & $7(13,2)$ & \\
\hline \multicolumn{4}{|l|}{ Doença de base } \\
\hline Neoplasias & $20(71,4)$ & $14(26,4)$ & \\
\hline Processos nflamatórios/infecciosos & $5(17,9)$ & $13(24,5)$ & \\
\hline Estenoses/obstruções/protrusões & $0(0)$ & $11(20,8)$ & $0,001 *$ \\
\hline Processos vasculares & $2(7,1)$ & $7(13,2)$ & \\
\hline $\begin{array}{l}\text { Processos patológicos de outra } \\
\text { natureza }\end{array}$ & $1(3,6)$ & $8(15,1)$ & \\
\hline \multicolumn{4}{|l|}{ Valores de hemoglobina mais baixos } \\
\hline Menor ou igual a 8 g/dl & $1(3,6)$ & $8(15,1)$ & 0,234 \\
\hline Maior que $8 \mathrm{~g} / \mathrm{dl}$ & $15(53,6)$ & $25(47,2)$ & \\
\hline Dado ausente & $12(42,8)$ & $20(37,7)$ & \\
\hline \multicolumn{4}{|l|}{ Valores de hematócrito mais baixos } \\
\hline Menor ou igual a 0,3 & $7(25,0)$ & $20(37,7)$ & 0,323 \\
\hline Maior que 0,3 & $10(35,7)$ & $20(37,7)$ & \\
\hline Dado ausente & $11(39,3)$ & $13(24,6)$ & \\
\hline \multicolumn{4}{|l|}{ Tipos de cirurgia } \\
\hline Ressecções/enucleações & $22(78,6)$ & $29(54,7)$ & \\
\hline $\begin{array}{l}\text { Plastias/reconstruções/desconexões/ } \\
\text { derivações }\end{array}$ & $4(14,3)$ & $12(22,6)$ & 0,143 \\
\hline Cirurgias exploratórias & $1(3,6)$ & $8(15,1)$ & \\
\hline Cauterizações/escleroses & $1(3,6$ & $4(7,5)$ & \\
\hline \multicolumn{4}{|l|}{ Motivos transcirúrgicos } \\
\hline Sim & $4(14,3)$ & $1(1,9)$ & $0,031 *$ \\
\hline Não & $24(85,7)$ & $52(98,1)$ & \\
\hline
\end{tabular}

*p estatístico significativo $\leq 0,05$ transfusionais. Não foram feitas associações entre TCH com valores de hemoglobina inferiores a $8 \mathrm{~g} / \mathrm{dl}$ devido à ausência de citações na maioria dos casos. Não houve associação estatística entre a prática de TCH com valores de hematócrito inferior ou igual a 0,30 no pós-cirúrgico. A Tabela 3 descreve as relações entre a prática da $\mathrm{TCH}$ no pós-cirúrgico e as variáveis categóricas correlatas. Não houve relação estatística entre as variáveis analisadas e a TCH.

\section{Discussão}

A prática de TCH é amplamente realizada mesmo em procedimentos eletivos onde sua indicação poderia ser mais restrita. ${ }^{(12-14)}$ Em nosso estudo observamos que, mesmo em cirurgias eletivas do aparelho digestivo, $47 \%, 34,6 \%$ e $45,7 \%$ dos pacientes receberam TCH nos períodos pré, trans e póscirúrgico, respectivamente. Embora a natureza desse estudo não permita inferir relações de causa e efeito, a taxa de mortalidade pós-cirúrgica nesses procedimentos foi elevada (11 casos). Existem evidências claras que a TCH pode resultar em pior prognóstico para pacientes críticos e que a perda de eficácia das hemácias está relacionada com tempo de estocagem, níveis de ácido nítrico ligados à hemoglobina livre, leucócitos do doador presentes no hemocomponente, resposta inflamatória do receptor e redução da plasticidade celular que influencia sua motilidade e trocas gasosas. ${ }^{(15)}$ Sabe-se, por exemplo, que o trauma perioperatório cirúrgico e o estresse podem ter um impacto importante na imunomodulação de pacientes com morbidades como neoplasias e que as transfusões alogênicas podem ainda potencializar a debilidade da resposta imune. ${ }^{(16)} \mathrm{Na}$ mesma linha desse achado, um estudo que lidou com 179 pacientes submetidos a cirurgias gástricas em decorrência de neoplasias, demonstrou, em sua análise univariada, um grande volume de sangue transfundido e associação da prática transfusional com um pior prognóstico. ${ }^{(17)} \mathrm{Um}$ outro estudo que analisou o impacto das TCH sobre 154 pacientes que foram submetidos a gastrectomias radicais evidenciou, através de análise multivariada, que a prática transfusional estava diretamente relacionada a menor sobrevida dos indivíduos operados quando comparados com pacientes que não receberam $\mathrm{TCH}$ $(154 \times 702){ }^{(18)}$

Ao mesmo tempo, o uso da eritropoetina recombinante humana associada com suplementação de ferro pode reduzir significativamente a necessidade de TCH perioperatórias, por elevar o hematócrito, em pacientes anêmicos portadores de neoplasias gastrointestinais. ${ }^{(19)}$ Quanto às estratégias voltadas para minimizar a perda sanguínea durante os procedimentos cirúrgicos podem ser citadas as técnicas de anestesia hipotensiva, manutenção da normotermia, dissecções cuidadosas, uso de cola de fibrina, eletrocoagulação, recuperação intraoperatória de células e hemodiluição. ${ }^{(6,18)} \mathrm{Com}$ relação às estratégias pós-cirúrgicas podem ser alistadas vigilância para detecção de quadros hemorrágicos, oxige- 
nação adequada, restrição de flebotomias para testes diagnósticos, recuperação pós-operatória de células, melhora da hemostasia por agentes farmacológicos, monitoramento da hipertensão, tolerância a anemia normovolêmica, uso meticuloso de anticoagulantes e agentes antiplaquetários. ${ }^{(6)}$

Existe grande variabilidade na prática transfusional entre instituições hospitalares diferentes, o que mostra que os parâmetros para indicação não são universais. Nesse estudo, viuse objetivamente que os valores hematológicos, status sistêmico do paciente e o tipo de procedimento cirúrgico realizado não serviram de parâmetro para se indicar a prática

Tabela 3. Relações entre a prática da TCH pós-cirúrgica (Transfusão de Concentrado de Hemácias) e as outras variáveis categóricas afins ( $\mathrm{n}=81)$

\begin{tabular}{|c|c|c|c|}
\hline \multirow[b]{2}{*}{ Variáveis } & \multicolumn{2}{|c|}{ TCH Pré-operatória } & \multirow{2}{*}{$\begin{array}{c}\text { Valor de } \\
\quad p\end{array}$} \\
\hline & $\begin{array}{l}\operatorname{Sim} \\
\mathrm{n}(\%)\end{array}$ & $\begin{array}{l}\text { Não } \\
\text { n (\%) }\end{array}$ & \\
\hline \multicolumn{4}{|l|}{ Gênero } \\
\hline Feminino & $15(40,5)$ & $19(43,2)$ & \multirow[t]{2}{*}{0,810} \\
\hline Masculino & $22(59,5)$ & $25(56,8)$ & \\
\hline \multicolumn{4}{|l|}{ Idade } \\
\hline Até 43 anos & $13(35,1)$ & $7(15,9)$ & \multirow[t]{3}{*}{0,119} \\
\hline De 44 a 56 anos & $10(27,1)$ & $13(29,6)$ & \\
\hline Acima de 56 anos & $14(37,8)$ & $24(54,5)$ & \\
\hline \multicolumn{4}{|l|}{ Tipo sanguíneo } \\
\hline A & $17(46,0)$ & $13(29,5)$ & \multirow[t]{4}{*}{0,057} \\
\hline $\mathrm{B}$ & $5(13,5)$ & $2(4,5)$ & \\
\hline $\mathrm{AB}$ & $0(0)$ & $2(4,5)$ & \\
\hline $\mathrm{O}$ & $15(40,5)$ & $27(61,4)$ & \\
\hline \multicolumn{4}{|l|}{ Fator RH } \\
\hline Positivo & $35(94,6)$ & $37(84,1)$ & \multirow[t]{2}{*}{0,122} \\
\hline Negativo & $2(5,4)$ & $7(15,9)$ & \\
\hline \multicolumn{4}{|l|}{ Instituição } \\
\hline 1 & $5(13,5)$ & $8(18,1)$ & \multirow[t]{4}{*}{0,908} \\
\hline 2 & $18(48,7)$ & $19(43,2)$ & \\
\hline 3 & $5(13,5)$ & $5(11,4)$ & \\
\hline 4 & $9(24,3)$ & $12(27,3)$ & \\
\hline \multicolumn{4}{|l|}{ Doença de Base } \\
\hline Neoplasias & $11(29,7)$ & $23(52,2)$ & \multirow{5}{*}{0,288} \\
\hline Processos inflamatórios/infecciosos & $10(27)$ & $8(18,2)$ & \\
\hline Estenoses/obstruções/protrusões & $7(18,9)$ & $4(9,1)$ & \\
\hline Processos vasculares & $4(10,8)$ & $5(11,4)$ & \\
\hline $\begin{array}{l}\text { Processos patológicos de outra } \\
\text { natureza }\end{array}$ & $5(13,5)$ & $4(9,1)$ & \\
\hline \multicolumn{4}{|l|}{ Tipos de cirurgia } \\
\hline Ressecções/enucleações & $19(51,4)$ & $32(72,7)$ & \multirow{4}{*}{0,147} \\
\hline $\begin{array}{l}\text { Plastias/reconstruções/ } \\
\text { desconexões/derivações }\end{array}$ & $11(29,7)$ & $5(11,4)$ & \\
\hline Cirurgias exploratórias & $5(13,5)$ & $4(9,1)$ & \\
\hline Cauterizações/escleroses & $2(5,4)$ & $3(6,8)$ & \\
\hline \multicolumn{4}{|l|}{ Valores de Hematócrito mais baixos } \\
\hline Menor ou igual a 0,3 & $21(56,8)$ & $16(36,4)$ & \multirow[t]{3}{*}{0,172} \\
\hline Maior que 0,3 & $9(24,3)$ & $14(31,8)$ & \\
\hline Dado ausente & $7(18,9)$ & $14(31,8)$ & \\
\hline \multicolumn{4}{|l|}{ Citação de motivo pós-cirúrgico } \\
\hline Sim & $14(37,8)$ & $11(25,0)$ & \multirow[t]{2}{*}{0,213} \\
\hline Não & $23(62,2)$ & $33(75,0)$ & \\
\hline
\end{tabular}

de TCH e que a escolha do profissional essencialmente norteou essa prática.

Um estudo realizado no Canadá, ${ }^{(20)}$ envolvendo 5.298 pacientes atendidos em unidades de terapia intensiva revelou que $25 \%$ receberam TCH. Variáveis independentes preditivas da prática transfusional foram avaliadas e incluíram idade, índice APACHE II (Acute Physiology And Chronic Health Evaluation), tipo de cirurgia e instituição de saúde. Após a análise multivariada ficou evidente que a variável institucional foi a mais relevante quando comparada com as outras citadas, corroborando os nossos achados pericirúrgicos. Foi verificado que $35 \%$ das transfusões sanguíneas (202 de 576 casos) foram realizadas em pacientes que apresentavam níveis de hemoglobina pré-transfusional variando de 9,5 g/dl a $10 \mathrm{~g} / \mathrm{dl}$ e em $80 \%$ desses casos foram solicitadas mais de duas unidades. A razão mais frequente para se administrarem as transfusões sanguíneas foi sangramento agudo (35\%) e disponibilização de oxigênio (25\%). Observou-se nesse estudo uma grande variabilidade na prática transfusional entre instituições e a adoção por muitos intensivistas do limiar $10 \mathrm{~g} / \mathrm{dl}$ de hemoglobina, que é um gatilho ultrapassado. ${ }^{(20,21)}$ Esses dados foram corroborados por outro estudo feito pelo mesmo grupo, evidenciando a grande variabilidade interinstitucional e intrainstitucional relacionada à prática transfusional em pacientes submetidos à cirurgia de bypass cardíaco. ${ }^{(5)}$ Outros estudos interinstitucionais realizados por grupos europeus também demonstraram grande variabilidade na prática transfusional em pacientes submetidos a cirurgias ortopédicas e de outras naturezas. ${ }^{(4,13,14)}$ Curiosamente, a literatura tem apontado associação entre TCH e manejo de neoplasias gástricas com pior prognóstico e menor sobrevida em dissonância com as práticas adotadas pelos profissionais durante as cirurgias no presente estudo. ${ }^{(17,18)}$

Mesmo havendo a citação de quadros de anemia como indicação dos procedimentos transfusionais no pré-cirúrgico e de relatos de hemorragia no pós-cirúrgico, a grande maioria dos casos transfundidos nesse estudo não apresentou justificativas para a sua realização. Acrescendo, vimos que não houve relação entre os valores de hemoglobina e hematócrito com a prática transfusional. As indicações dos procedimentos transfusionais não raro se embasam em critérios objetivos e subjetivos, como "gatilhos" variáveis, critérios semiotécnicos, instituição de origem, experiência profissional, condições clínicas e valores baixos de hemoglobina, muitas vezes sem sintomatologia clínica associada. ${ }^{(11,22-24)}$ As concentrações de hemoglobina podem ser facilmente variáveis e por anos conduziram os valores chamados de "gatilho transfusional"; todavia, os valores ideais de hemoglobina variam consideravelmente de um paciente para outro sendo norteados por variáveis múltiplas como idade, doença crônica presente (especialmente doenças de artéria coronária), diagnóstico atual, causa de anemia e tolerância a anemia. ${ }^{(15,25,26)}$ Nesse sentido, existem es- 
tudos que demonstram claramente boa tolerabilidade a anemia de pacientes com valores baixos de hemoglobina e a eficácia de procedimentos de conservação e estimulação da produção autóloga de hemácias, que maximizam o hematócrito final sem TCH. ${ }^{(27,28)}$ Um outro aspecto a ser considerado é que, em procedimentos cirúrgicos gástricos, por exemplo, a perda sanguínea pode ser amplamente minimizada através de técnicas cirúrgicas minimamente invasivas, tempo cirúrgico reduzido e planejamento cirúrgico envolvendo a preservação do sangue do paciente evitando quadros hemorrágicos iatrogênicos. $^{(18)}$

Dados essenciais referentes a valores de hemoglobina, hematócrito, ASA e assinatura de pacientes consentindo com o procedimento transfusional estavam muitas vezes ausentes dos prontuários médicos. Estudos prévios demonstraram a mesma deficiência em registro de dados, como uma análise onde houve a notação do motivo transfusional em apenas $23 \%$ dos casos. ${ }^{(4,14)}$ Um estudo realizado em um centro obstétrico no Reino Unido revelou que apenas 9\% dos pacientes consentiram com o procedimento transfusional, sabendo sobre os potenciais riscos do procedimento. Ao mesmo tempo, pacientes foram transfundidos, sem o reporte de um motivo no prontuário, provavelmente identificando a eleição do procedimento por critérios fundamentalmente pessoais do que através de parâmetros mensuráveis. ${ }^{(22)}$

\section{Conclusões}

Concluindo, o estudo revelou que a prática de $\mathrm{TCH}$ em cirurgias eletivas do aparelho digestivo foi heterogênea entre as instituições hospitalares avaliadas, careceu de valores laboratoriais específicos que norteassem a indicação da transfusão e se relacionou com a patologia de base mas não com o tipo de intervenção cirúrgica realizada. Esses elementos evidenciam a necessidade de se rediscutir a prática de TCH em pacientes cirúrgicos e de elaborarem programas de educação continuada entre os profissionais da saúde no que se referem às indicações das transfusões de hemocomponentes.

\section{Abstract}

The objective of this study was to understand the determinative factors for packed red cell transfusions in elective digestive tract surgeries in four hospital units in Montes Claros, Minas Gerais, Brazil. Retrospective analyses were performed by collecting data from the clinical records of patients submitted to elective digestive tract surgeries from June 2007 to December 2008 at these four hospital units. Eighty-one cases (42\% females and 58\% males) were included in this study. The average age was 55.5 years old (Standard deviation \pm 19.11 years). The commonest reported underlying disease was neoplasia (42\%). Of these patients, 38 (47\%) were transfused in the immediate preoperative period, 28 (34.6\%) during the surgery and 37 (45.7\%) in the immediate postoperative period. In the univariate analysis, there was an association between the data of transfusions and the different institutions. In the surgical phase, there were correlations between transfusion and the reason for transfusion, RH factor (Rhesus), institutional policy and the underlying disease. In the postoperative phase, no association was identified. In all phases, there were not correlations between the type of surgical procedures, ASA index (American Society of Anesthesiology), and hemoglobin and hematocrit values. Therefore, this study demonstrated that the blood transfusions in elective digestive tract surgeries were heterogeneous between hospitals and were not related to specific laboratorial values; they were associated to the underlying pathology, but not with the type of surgical procedure and generally followed the criteria of the healthcare specialist.

Keywords: Digestive system surgical procedures; Erythrocyte transfusion; Blood componente transfusion

\section{Agradecimentos}

À Prof. Marise Silveira pelo tratamento estatístico è à Fapemig pelo apoio financeiro.

\section{Referências}

1. Zago MA, Passeto Falcão R, Pasquita R. Hematologia fundamentos e prática. Atheneu: Rio de Janeiro; 2001.

2. Alvarez G, Hebert PC, Szick S. Debate: transfusing to normal haemoglobin levels will not improve outcome. Crit Care. 2001; 5(2):56-63.

3. Carson JL, Hill S, Carless P, Hebert P, Henry D. Transfusion triggers: a systematic review of the literature. Transfus Med Rev. 2002; 16(3):187-9.

4. Drouet N. European transfusion practices: the SANGUIS survey. Cah Anesthesiol. 1994;42(3):425-8.

5. Shehata N, Wilson K, Mazer CD, Tomlinson G, Streiner D, Hebert $\mathrm{P}$, et al. The proportion of variation in perioperative transfusion decisions in Canada attributable to the hospital. Can J Anaesth. 2007;54(11):902-7.

6. Goodnough LT, Shander A, Spence R. Bloodless medicine: clinical care without allogeneic blood transfusion. Transfusion. 2003; 43(5):668-76.

7. Chigbu B, Onwere S, Kamanu C, Aluka C, Okoro O, Feyi-Waboso $\mathrm{P}$, et al. Lessons learned from the outcome of bloodless emergency laparotomies on Jehovahs Witness women presenting in the extremis with ruptured uterus. Arch Gynecol Obstet. 2009;279 (4):469-72.

8. Pompei E, Tursi V, Guzzi G, Vendramin I, Ius F, Muzzi R, Auci E, et al. Mid-term clinical outcomes in cardiac surgery of Jehovahs witnesses. J Cardiovasc Med (Hagerstown). 2009 Nov 19. [Epub ahead of print].

9. Ball AM, Winstead PS. Recombinant human erythropoietin therapy in critically ill Jehovahs Witnesses. Pharmacotherapy. 2008; 28(11):1383-90.

10. Rottman G, Ness PM. Acute normovolemic hemodilution is a legitimate alternative to allogeneic blood transfusion. Transfusion. 1998;38(5):477-80.

11. Maddux FW, Dickinson TA, Rilla D, Kamienski RW, Saha SP, Eales $\mathrm{F}$, et al. Institutional variability of intraoperative red blood cell utilization in coronary artery bypass graft surgery. Am J Med Qual. 2009;24(5):403-11. 
12. Rao SV, Chiswell K, Sun JL, Granger CB, Newby LK, Van de Werf $\mathrm{F}$, et al. International variation in the use of blood transfusion in patients with non-ST-segment elevation acute coronary syndromes. Am J Cardiol. 2008;101(1):25-9.

13. Pedersen AB, Mehnert F, Overgaard S, Møller B, Johnsen SP. Transfusion practice in total hip arthroplasty in Danish departments of orthopaedic surgery. Ugeskr Laeger. 2009;171(12):973-7.

14. Ten Duis HJ, van Dalen KC, Giovanetti AM, Smit Sibinga CT, Sirchia G. The use of blood and blood products in total hip arthroplasty in 31 European hospitals, 1990/91. Ned Tijdschr Geneeskd. 1996;140(25):1307-12.

15. Hajjar LA, Auler-Junior JO, Santos L, Galas F. Blood tranfusion in critically ill patients: state of the art. Clinics. 2007;62(4):507-24.

16. Chen G, Zhang FJ, Gong M, Yan M. Effect of perioperative autologous versus allogeneic blood transfusion on the immune system in gastric cancer patients. J Zhejiang Univ Sci B. 2007; 8(8):560-5.

17. Bellantone R, Sitges-Serra A, Bossola M, Doglietto GB, Malerba M, Franch G, et al. Transfusion timing and postoperative septic complications after gastric cancer surgery: a retrospective study of 179 consecutive patients. Arch Surg. 1998;133(9):988-92.

18. Ojima T, Iwahashi M, Nakamori M, Nakamura M, Naka T, Katsuda $\mathrm{M}$, et al. Association of allogeneic blood transfusions and longterm survival of patients with gastric cancer after curative gastrectomy. J Gastrointest Surg. 2009;13(10):1821-30.

19. Braga M, Gianotti L, Vignali A, Gentilini O, Servida P, Bordignon $\mathrm{C}$ et al. Evaluation of recombinant human erythropoietin to facilitate autologous blood donation before surgery in anaemic patients with cancer of the gastrointestinal tract. Br J Surg. 1995; 82(12):1637-40

20. Hebert PC, Wells G, Martin C, Tweeddale M, Marshall J, Blajchman $\mathrm{M}$, et al. Variation in red cell transfusion practice in the intensive care unit: a multicentre cohort study. Crit Care. 1999;3(2):57-63.
21. Carson JL, Hill S, Carless P, Hebert P, Henry D. Transfusion triggers: a systematic review of the literature. Transfus Med Rev. 2002;16(3):187-9.

22. Parker J, Thompson J, Stanworth S. Parker J, Thompson J, Stanworth $\mathrm{S}$. A retrospective one-year single-centre survey of obstetric red cell transfusions. Int J Obstet Anesth. 2009;18(4):309-13.

23. Dos Santos AM, Guinsburg R, Procianoy RS, Sadeck LD, Netto AA, Rugolo LM, et al. Variability on red blood cell transfusion practices among Brazilian neonatal intensive care units. Transfusion. 2009 Aug 26. [Epub ahead of print].

24. Morley SL. Red blood cell transfusions in acute paediatrics. Arch Dis Child Educ Pract Ed. 2009;94(3):65-73.

25. Hardy JF. Should we reconsider triggers for red blood cell transfusion? Acta Anaesthesiol Belg. 2003;54(4):287-95.

26. Vincent JL, Baron JF, Reinhart K, Gattinoni L, Thijs L, Webb A, et al. Anemia and blood transfusion in critically ill patients. JAMA. 2002;288(12):1499-507. Comment in: JAMA. 2002 Sep 25;288 (12):1525-6. JAMA. 2003 Mar 12;289(10):1242. JAMA. 2003 Mar 12;289(10):1242-3; author reply 1243. JAMA. 2003 Mar 12;289(10):1242.

27. Berend K, Levi M. Management of adult Jehovahs witness patients with acute bleeding. Am J Med. 2009;122(12):1071-6.

28. Oh SY, Kim SH, Kwon HC, Lee S, Kim KH, Kwon KA, et al. Bloodless cancer treatment results of patients who do not want blood transfusion: single center experience of 77 cases. Support Care Cancer. 2009 Oct 20. [Epub ahead of print].

Recebido: $24 / 2 / 2010$

Aceito: $23 / 6 / 2010$ 